\title{
PROPOSAL FOR EXPERIMENTS ON WAVE FUNCTION LOCALISATION
}

\author{
V.Danilov, A.Aleksandrov, ORNL SNS Project, Oak Ridge, TN
}

\begin{abstract}
Well-known manifestations of quantum effects in accelerators are related to the quantum fluctuations of synchrotron radiation and the spin polarisation. There are more possibilities to see quantum mechanics signatures in accelerators. It is shown that Bell's inequalities for nonidentical particles, as well as the wave function localisation due to measurement, could be tested with conventional accelerator components and techniques.
\end{abstract}

\section{INTRODUCTION}

This work was motivated by papers of Vinokurov and his colleagues [1]. They presented studies of one electron trajectories in a storage ring in presence of strong synchrotron radiation, and confirmed the randomness of its motion. The experiments showed that the electron wave function didn't spread out over the ring but, instead, its the localisation is fairly good and "the possible cause of the localisation is the interaction with the radiation field, which acts like a continuos measurement". Thus, the nontrivial question is how and when the localisation occurs and is it related to the measurement (or equivalent) process.

The quantum mechanical behaviour of particles could be perfectly demonstrated by interference pattern, appearing when particles pass through a double-slit and detected at the screen behind it. For two particles, having correlated spins (or other variables), there exist another exceptional possibility to measure, if the particle wave functions are coupled (entangled) to each other. In $70^{\text {th }}$ John Bell [2] presented a scheme for polarisation measurement for two particles, which gives different results for some correlation function (S) of particle spins, if measured on various axes, in case when two particle spins are entangled or independent. The function $S$, as well as three related examples, is described in appendix of the full variant of this paper [3]. The paper deals with the simple set-up to check the entanglement of the photons and to check if the process of conversion of polarised photon into a polarised electron breaks the entanglement.

\section{IS SUPERLUMINAL COMMUNICATION POSSIBLE?}

In order to show what type of experiments could be done with entangled photons, we present an experimental scheme that deals with possibility to transmit signals faster than the speed of light.
Let's take the source of entangled photons, moving in opposite directions, having equal (entangled or coupled to each other) polarisation. For every photon wave function consists of combination of parallel and perpendicular polarisation, with the wave function $\psi$

$$
\psi \propto\left|1_{\perp}, 2_{\perp}\right\rangle+\left|1_{\|}, 2_{\|}\right\rangle,
$$

where symbols $\perp$ and $\|$ mean perpendicular and parallel polarisation with respect to some axis. ${ }^{1}$ One can see that this wave function can not be factorised and the photons in this state are called entangled photons. The remarkable feature of this state is that if one photon polarisation is measured to be equal to some value (e.g. angle in radians with respect to some axis), the opposite photon instantly has the same polarisation.

Figure 1 shows the simplest experimental set-up with the future experimental outcome to be a puzzle from theoretical point of view. The scheme consists of two parts.

The right part is just polarisation measurement scheme, which consists of polarisation splitter cube and two Photo Multipliers (PMT's) to count the photons, and the switch, which either deflects the beam from the cube or directs the beam into it. We assume that the deflected beam is travelling without measurement. But if the photon passes through the cube, its polarisation is measured with $100 \%$ efficiency.

The left part is an interferometer (the principal scheme is taken from [4]. The photon in state (1), travelling left, is splitted in the cube with parallel polarisation transmitted through it and perpendicular reflected down. We assume that the reflection in halftransparent mirror shifts the phase of the light to quarter of oscillation, the regular mirrors and polarisation splitter for the perpendicular polarisation change the oscillation phase to half, and each direct passing of light through them leaves the phase unchanged. In addition, we assume that the phase shifter below the cube shifts the oscillation phase to $-\pi / 2$. Parallel polarisation is transformed into perpendicular after the upper left mirror in order to have interference pattern for both photon paths. It easy to calculate the phase difference for both photon paths and find that, if the photon polarisation is not measured at the right shoulder of the set-up, the Photo Multiplier A (PMT $\mathrm{A}$ in the scheme) has zero phase difference between two photon paths ${ }^{2}$. Thus it has nonzero signal, while the PMT $\mathrm{B}$ has $\pi$ phase advance and no signal.

\footnotetext{
${ }^{1}$ Therefore the polarisation of every photon, if measured separately, is equal for any axis

2 The upper left path has reflection-related phase shift in PMT A equal to $\pi$ (due to the upper left mirror) $+\pi / 2$ (due to the half-transparent
} 
The described above scheme gives interference if the photon is not measured in the right side PMT's. If it is not deflected by a fast switch and its polarisation is measured, it automatically travels through one path (which correlates with the results of the measurements on the right), and both PMT A and PMT B have statistically equal signals. Thus if the collapse of two photon wave function is instant, we can transmit signals faster than the speed of light at the statistical level.

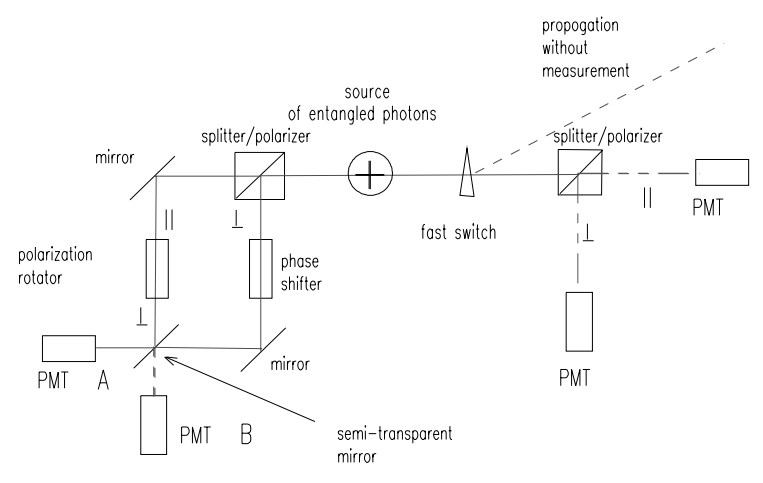

Figure 1 Experimental set-up with interference pattern to disappear at the left if the polarization measured at the right.

\section{DOES THE CATHODE BREAK THE ENTANGLEMENT?}

Let's take the source of entangled photons similar to the described in Section 1. Figure 2 shows proposed experimental set-up to measure if the absorption of the photon and related to it emission of the electron breaks the entanglement with another photon. If taken without the cathode, the set-up resembles one use by Alain Aspect and his colleagues [5]. The entangled photons after splitting in polarimeter cubes are measured by photomultipliers (PMT) at the left and converted to polarised electrons at the right, which are measured by Mott polarimeter Microchannel Plates (MCPs). Cube I directs different polarisations into two photon channels. These photons are transformed into circular photons and produce electrons with longitudinal polarisations (forward or backward, depending on the polarisation of the incident photons). The spin rotator converts the longitudinal polarisation into a transverse one. Therefore measured electron spin "up" corresponds to the photon with the parallel polarisation, and spins "down" - to the perpendicular polarisation.

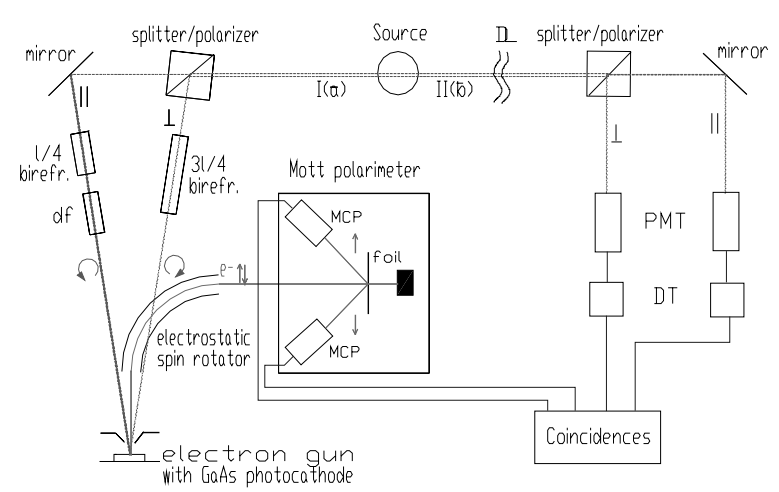

Figure 2 Proposed experimental setup. Two polarimeters $I$, in orientation a, and II, in orientation b, split the first photon to produce polarized electrons (at the left part) and to direct the second one to two photomultipliers (at the right side). Each polarimeter is rotatable around the axis of the beam.

\section{POSSIBLE SCHEME TO CHECK THE EQUIVALENCE BETWEEN RADIATION AND MEASUREMENT}

Finally, we come to the questions of paper [1]. The studies, presented in it, show one-electron trajectory in a storage ring in presence of strong synchrotron radiation. The experiments showed that the electron wave function localisation is fairly good. Is this localisation related to the measurement process? Or, in reformulated form, when the electron radiate, when does its entanglement with the photon break?

It turns out to be that there exists already optical scheme for accelerators (namely, ATF in KEK, Japan) to measure sizes of the electron beam with the interferogram [6]. Figure 3 shows the rough scheme of the experiment.

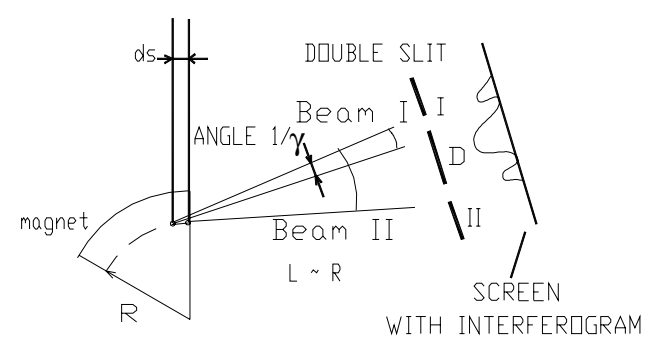

Figure 3 Principal scheme of the SR experiment

The circles at the Figure 3 show two consecutive positions of an electron. It radiates synchrotron radiation into angle $1 / \gamma$. The double slit is placed perpendicularly to the Synchrotron Radiation (SR) light. Light Beam I and Light Beam II represent two different situations. Beam I mirror $)=3 \pi / 2$. The lower path has the phase shift $\pi$ (due to the cube) $-\pi / 2$ (due to the phase shifter) $+\pi$ (due to the mirror) $=3 \pi / 2$, therefore the phase shifts are equal. Similar calculation gives $\pi$ phase difference between two paths and, therefore, no signal in PMT B. 
corresponds to the situation when the SR light breaks away from the electron at the short distance (the act equivalent to measurement happened). The width at the figure is $1 / \gamma$ as if the act of radiation occurred instantly. If the width of the beam is less than the distance D between the slits, the interference pattern is absent. Beam II represents the classical situation when the radiation field is running along with the electron. In this case both slits see approximately the same intensity (at the same time, because the electron is ultrarelativistic and its trajectory is perpendicular to the slits) and produce the interferogram.

Paper [6] has presented the results for the visibility versus the distance $\mathrm{D}$ between slits. Roughly, the interferogram disappears when this distance is about 30 $\mathrm{mm}$. The authors relate this fact to the horizontal beam size, which was calculated to be equal to $39 \mu \mathrm{m}$.

These number impose some limitations on the length when the photon become independent of the electron. First of all, if the process of radiation happens instantly, the size of the light spot would be about $\mathrm{L} / \gamma \approx 2 \mathrm{~mm}$ (for $\mathrm{R}$ $\approx 6 \mathrm{~m}, \mathrm{~L} \approx 7 \mathrm{~m}, \gamma \approx 3000$ ). Since the interferogram disappear for much larger distances $(30 \mathrm{~mm})$, this possibility is ruled out. If we assume that the beam actual size is much smaller than $39 \mu \mathrm{m}$, and the interference pattern disappear because of the photon wave function localisation, the distance of this ("measurement-like") process is about 30 $\mathrm{mm}$. This could be regarded as the lower limit for the "measurement" distance for the ATF set of parameters.

We think that it is of a great interest to combine both [1] and [6] experiments, to measure the distance of the photon localisation process, the "size" of one electron, etc.

\section{CONCLUSION}

Three schemes to check when the quantum measurement happens are presented. All look realisable with modern accelerators or their components.

\section{ACKNOWLEDGMENTS}

The authors wish to thank N. Vinokurov, Yu. Efremenko, Yu. Kamyshkov, S. Ovchinnikov, L. Okun' for fruitful discussions and G. Bitton, W.Griece, C. D'Helon for their interest to the paper and pointing out the similarity of the first scheme to a quantum eraser. Research sponsored by the DOE, under contract no. DEAC05-00OR22725with UT-Batelle, LLC for ORNL

\section{REFERENCES}

[1] N. Vinokurov, "Longitudinal Motion in Storage Rings and Quantum Excitation", Proceedings of Joint Accelerator School, Montreux, and CERN, Switzerland, 11-20 May, 1998. I. Pinayev et al, NIM A 375 (1996) 7173.

[2] J.S. Bell, Physics (N.Y.) v 1, 195 (1965)

[3] V. Danilov and A. Aleksandrov, "Proposal for Experiments on Wave Function Localisation" http://www.sns.gov/APGroup/Papers/TechMemos/TechM emos.html

[4] R. Penrose, "Shadows of the mind", Oxford University Press, Oxford, 1994. p. 261-262

[5] A. Aspect, et. al., Phys. Rev. Lett., v 49, n 2, (1982)

[6] T. Mitsuhashi and T. Naito, $6^{\text {th }}$ EPAC, Stockholm, Sweden, (1998) p. 1565 